\title{
A five-equation dissipative model for the simulation of two-phase flows
}

\author{
M. Labois ${ }^{1}$, H. Guillard ${ }^{2}$ \& M. Grandotto ${ }^{1}$ \\ ${ }^{1}$ DEN/DER/SSTH/LMDL, CEA Cadarache, France \\ ${ }^{2}$ Projet SMASH, INRIA Sophia-Antipolis, France
}

\begin{abstract}
This work deals with the design of a five-equation dissipative model for the simulation of two-phase flows and its numerical approximation. Two-phase flows are usually modeled using the well-known one-pressure two-velocity model, which is not hyperbolic, or using a two-pressure two-velocity model, whose complexity makes it costly. Finally, five-equation reduced models can be used, but they feature a unique velocity, which makes them unable to reproduce complex flows.

Our five-equation dissipative model is derived from the standard six-equation bifluid model using the Chapman-Enskog expansion technique. Developments to the first order lead to a hyperbolic system, and even if the model features only one velocity, dissipative second-order terms enable it to deal with velocity disequilibria.

A finite-volume numerical approximation of this model using a fractional step approach is proposed. After the convective step, which takes into account the hyperbolicity of the convective part, the solution of the dissipative step is evaluated. Numerical tests are presented, where the capability of the model to deal with flow featuring phenomena due to velocity disequilibria is shown.

Keywords: two-phase flows, velocity disequilibria, Chapman-Enskog expansion, finite-volume scheme.
\end{abstract}

\section{Introduction}

Two-phase flows arise in a large range of applications, and their numerical simulations are still an issue. Mixture models are often used for this purpose. But there are situations where velocity disequilibria between the phases have to be 
taken into account and for these cases, the most widespread model is the bifluid model based on the assumption of the phase pressure equality [1], which feature six equations in one dimension. This model is however basically non-hyperbolic, which can lead to numerical failures.

Thus, seven-equation models [2] have been proposed, with the addition of an equation on the volume fraction. This ensures the hyperbolicity, but it is seldom used because of its computational cost. Finally, five-equation models exist [3], which includes unique pressure and velocity for both phases, but this feature prevent them to model complex flows.

Here, we discuss a model that has been obtained using a Chapman-Enskog expansion [4] of the six-equation model. This gives us the five-equation model when the expansion is performed at the order zero, but expansion at the second order gives additional terms that can model the difference between phase velocities. The derivation of this model will be presented in the next section, then we will show some of its properties. Next, we will discuss its numerical approximation and present eventually some numerical results.

\section{Derivation of a five-equation model with additional second-order terms}

Our model is derived using a Chapman-Enskog expansion [4] of the classical bifluid model, which can be written

$$
\begin{aligned}
& \partial_{t} \alpha_{k} \rho_{k}+\operatorname{div}\left(\alpha_{k} \rho_{k} \underline{u}_{k}\right)=0 \\
& \partial_{t} \alpha_{k} \rho_{k} \underline{u}_{k}+\operatorname{div}\left(\alpha_{k} \rho_{k} \underline{u}_{k} \otimes \underline{u}_{k}\right)+\alpha_{k} \nabla p=\underline{M}_{k}^{d}+\alpha_{k} \rho_{k} \underline{g} \\
& \partial_{t} \alpha_{k} \rho_{k} e_{k}+\operatorname{div}\left(\alpha_{k}\left(\rho_{k} e_{k}+p\right) \underline{u}_{k}\right)=-p \partial_{t} \alpha_{k}+\underline{u}_{I} \cdot \underline{M}_{k}^{d}+\alpha_{k} \rho_{k} \underline{u}_{k} \cdot \underline{g}
\end{aligned}
$$

where $\alpha_{k}$ are the phase volume fractions and verify $\alpha_{1}+\alpha_{2}=1, \rho_{k}$ are the phase densities, $\underline{u}_{k}$ the phase velocities, $p$ the pressure and $e_{k}$ the phase total specific energies, with $e_{k}=i_{k}+\underline{u}_{k}^{2} / 2, i_{k}$ being the phase internal specific energies. $g$ stands for the gravity. The velocity at the interface is modeled by $\underline{u}_{I}=Y_{1} \underline{u}_{1}+Y_{2} \underline{u}_{2}$, and the drag force terms are written $\underline{M}_{k}^{d}=(-1)^{k+1} \frac{\rho}{\varepsilon_{u}}\left(\underline{u}_{2}-\underline{u}_{1}\right)$, where we have introduced the mixture density $\rho=\alpha_{1} \rho_{1}+\alpha_{2} \rho_{2}$ and the mass fraction $Y_{k}=\frac{\alpha_{k} \rho_{k}}{\rho}$. $\varepsilon_{u}$ denotes the characteristic time of the velocity relaxation, and is supposed to be small.

An expansion to the order zero in $\varepsilon_{u}$ gives the five-equation model [3] that can be written

$$
\begin{aligned}
& \partial_{t}\left(\alpha_{1} \rho_{1}\right)+\operatorname{div}\left(\left(\alpha_{1} \rho_{1}\right) \underline{u}\right)=0 \\
& \partial_{t}\left(\alpha_{2} \rho_{2}\right)+\operatorname{div}\left(\left(\alpha_{2} \rho_{2}\right) \underline{u}\right)=0 \\
& \partial_{t} \rho \underline{u}+\operatorname{div}(\rho \underline{u} \otimes \underline{u})+\nabla p=\rho \underline{g} \\
& \partial_{t} \rho e+\operatorname{div}((\rho e+p) \underline{u})=0 \\
& \left(\alpha_{1} C_{2}+\alpha_{2} C_{1}\right)\left(\partial_{t} \alpha_{1}+\underline{u} . \partial_{x} \alpha_{1}\right)+\alpha_{1} \alpha_{2}\left(C_{1}-C_{2}\right) \operatorname{div} \underline{u}=0
\end{aligned}
$$


where $\rho e=\rho i+\underline{u}^{2} / 2$ is the total energy of the mixture, with $\rho i=\alpha_{1} \rho_{1} i_{1}+\alpha_{2} \rho_{2} i_{2}$ the internal energy of the mixture and $C_{k}=\rho_{k} c_{k}^{2}$ is the adiabatic bulk modulus of phase $k$, with $c_{k}$ the speed of sound.

If we now perform a first order expansion, we obtain an expression of the relative velocity between the phases as a function of the state variables. More precisely, it can be written $\underline{u}_{r}=\left(\underline{u}_{1}-\underline{u}_{2}\right)=\varepsilon_{u} \frac{\left(Y_{1}-\alpha_{1}\right)}{\rho} \nabla p$. Introducing this expression in system (1), we obtain the following system with additional second-order terms

$$
\begin{aligned}
& \partial_{t}\left(\alpha_{1} \rho_{1}\right)+\operatorname{div}\left(\left(\alpha_{1} \rho_{1}\right) \underline{u}\right)=-\operatorname{div}\left(\rho Y_{1} Y_{2} \underline{u}_{r}\right) \\
& \partial_{t}\left(\alpha_{2} \rho_{2}\right)+\operatorname{div}\left(\left(\alpha_{2} \rho_{2}\right) \underline{u}\right)=\operatorname{div}\left(\rho Y_{1} Y_{2} \underline{u}_{r}\right) \\
& \partial_{t} \rho \underline{u}+\operatorname{div}(\rho \underline{u} \otimes \underline{u})+\nabla p=\rho \underline{g} \\
& \partial_{t} \rho e+\operatorname{div}((\rho e+p) \underline{u})=\operatorname{div}\left(\left(h_{2}-h_{1}\right) \rho Y_{1} Y_{2} \underline{u}_{r}\right)+\rho \underline{u} \cdot \underline{g} \\
& \left(\alpha_{1} C_{2}+\alpha_{2} C_{1}\right)\left(\partial_{t} \alpha_{1}+\underline{u} \cdot \partial_{x} \alpha_{1}\right)+\alpha_{1} \alpha_{2}\left(C_{1}-C_{2}\right) \operatorname{div} \underline{u} \\
& \quad=\left(Y_{1} \alpha_{1} C_{2}-Y_{2} \alpha_{2} C_{1}\right) \underline{u}_{r} \cdot \nabla \alpha_{1}-\alpha_{1} \alpha_{2}\left(C_{1} \operatorname{div}\left(Y_{2} \underline{u}_{r}\right)+C_{2} \operatorname{div}\left(Y_{1} \underline{u}_{r}\right)\right) \\
& \quad-\left(\alpha_{1} \alpha_{2}+\left(\frac{\alpha_{1} Y_{1}}{\rho_{2} \kappa_{2}}-\frac{\alpha_{2} Y_{2}}{\rho_{1} \kappa_{1}}\right)\left(Y_{1}-\alpha_{1}\right)\right) \underline{u}_{r} . \nabla p
\end{aligned}
$$

where $h_{k}=i_{k}+p / \rho_{k}$ is the specific enthalpy of phase $k$ and $\kappa_{k}=\left(\frac{\partial i_{k}}{\partial p}\right)_{k}$. In the following, we will assume that this system of equations is completed with Stiffened gas equations of state for both phase, i.e. $i_{k}$ is such that $i_{k}=\frac{p+\gamma_{k} p_{k, \infty}}{\left(\gamma_{k}-1\right) \rho_{k}}+i_{k, 0}$, with $\gamma_{k}, p_{k, \infty}$ and $i_{k, 0}$ are constants describing the thermodynamical properties of the material.

\section{Properties of the model}

The five-equation model (2), and therefore the model with second-order terms (3), is an unconditionally hyperbolic model, as shown by Murrone and Guillard [3]. Indeed, it features three distinct real eigenvalues, whose associated eigenvectors span $\mathbb{R}^{5}$. These eigenvalues are given by $u, u-\hat{c}$ and $u+\hat{c}$, where $\hat{c}$ is the wellknown speed of sound in a multiphase flow given by the equation $1 /\left(\rho \hat{c}^{2}\right)=$ $\alpha_{1} /\left(\rho_{1} c_{1}^{2}\right)+\alpha_{2} /\left(\rho_{2} c_{2}^{2}\right)[5]$.

A second very interesting property of this model is the existence of two independent entropy-entropy flux pairs, one for each phase, which are consistent with the additional second-order terms. These pairs are $\left(-\alpha_{k} \rho_{k} s_{k},-\alpha_{k} \rho_{k} s_{k} u\right)$, where $s_{k}$ is the specific entropy of phase $k$.

Indeed, algebraic manipulations of the system (3) gives two equation on the phase entropies $s_{k}$

$$
\begin{aligned}
& -\partial_{t}\left(\alpha_{k} \rho_{k} s_{k}\right)-\operatorname{div}\left(\alpha_{k} \rho_{k} s_{k} \underline{u}\right) \\
& \quad=\varepsilon_{u} \operatorname{div}\left((-1)^{k+1} Y_{k} Y_{k^{\prime}} s_{k}\left(Y_{1}-\alpha_{1}\right) \nabla p\right)-\varepsilon_{u} \frac{\rho Y_{k^{\prime}}}{T_{k}}\left(\frac{\left(Y_{1}-\alpha_{1}\right)}{\rho}\right)^{2}\|\nabla p\|^{2}
\end{aligned}
$$

where $k^{\prime}=3-k$. Considering equation (4), it is therefore clear that dissipative terms due to velocity disequilibrium increase the phase entropies along the 
trajectories. As a consequence, the mixture entropy $s=Y_{1} s_{1}+Y_{2} s_{2}$ is also an increasing variable along the trajectories.

\section{Numerical approximation}

A fractional step is performed to solve numerically the system (3), as performed by Guillard and Duval [6]. This consists in first solving the convective step, i.e. solving the classical five-equation system (2), and then focusing on equations where only second-order dissipative terms appear.

\subsection{Convective step}

To solve the convective step, we use the Finite Volume method proposed by Murrone and Guillard [3]. This method is based on VFRoe-ncv methods [7], but characteristics on both sides of the discontinuity are linearized when using the approximate Riemann solver, which leads to a more robust solver than classical VFRoe-ncv methods.

It should be noted that the equation on the phase 1 volume fraction is not solved using the form given in system (2), because this form may cause numerical instabilities when cohesion pressures $p_{k, \infty}$ are very different, which is usually the case when dealing with gas-liquid flows. Indeed, when discretizing this equation, large rounding errors arise when evaluating the pressure. Thus, the volume fraction equation is transformed into

$$
\partial_{t} \alpha_{1}+\nabla .\left(\alpha_{1} \underline{u}\right)-\frac{\alpha_{1} C_{2}}{\alpha_{1} C_{2}+\alpha_{2} C_{1}} \operatorname{div} \underline{u}=0
$$

which prevents the propagation of large rounding errors.

\subsection{Dissipative step}

The dissipative step consists in solving the following equations, using the state $\operatorname{vector}^{t}\left(\rho Y, \rho e, \alpha_{1}, \rho, \rho \underline{u}\right)$

$$
\begin{aligned}
& \partial_{t} \rho Y_{1}=-\operatorname{div}\left(\rho Y_{1} Y_{2} \underline{u}_{r}\right) \\
& \partial_{t} \rho e=\operatorname{div}\left(\left(h_{2}-h_{1}\right) \rho Y_{1} Y_{2} \underline{u}_{r}\right) \\
& \left(\alpha_{1} C_{2}+\alpha_{2} C_{1}\right) \partial_{t} \alpha_{1}=\left(Y_{1} \alpha_{1} C_{2}-Y_{2} \alpha_{2} C_{1}\right) \underline{u}_{r} . \nabla \alpha_{1} \\
& -\alpha_{1} \alpha_{2}\left(C_{1} \operatorname{div}\left(Y_{2} \underline{u}_{r}\right)+C_{2} \operatorname{div}\left(Y_{1} \underline{u}_{r}\right)\right) \\
& -\left(\alpha_{1} \alpha_{2}+\left(\frac{\alpha_{1} Y_{1}}{\rho_{2} \kappa_{2}}-\frac{\alpha_{2} Y_{2}}{\rho_{1} \kappa_{1}}\right)\left(Y_{1}-\alpha_{1}\right)\right) \underline{u}_{r} . \nabla p \\
& \partial_{t} \rho=0 \\
& \partial_{t} \rho \underline{u}=0
\end{aligned}
$$


Because the mixture density $\rho$ and momentum $\rho \underline{u}$ are constant with respect to time during the dissipative step, the system can be simplified and written with the variables ${ }^{t}\left(Y_{1}, i, \alpha_{1}\right)$

$$
\begin{aligned}
& \rho \partial_{t} Y_{1}=-\operatorname{div}\left(\rho Y_{1} Y_{2} \underline{u}_{r}\right) \\
& \rho \partial_{t} i=\operatorname{div}\left(\left(h_{2}-h_{1}\right) \rho Y_{1} Y_{2} \underline{u}_{r}\right) \\
& \left(\alpha_{1} C_{2}+\alpha_{2} C_{1}\right) \partial_{t} \alpha_{1} \\
& \quad=\left(Y_{1} \alpha_{1} C_{2}-Y_{2} \alpha_{2} C_{1}\right) \underline{u}_{r} \cdot \nabla \alpha_{1}-\alpha_{1} \alpha_{2}\left(C_{1} \operatorname{div}\left(Y_{2} \underline{u}_{r}\right)+C_{2} \operatorname{div}\left(Y_{1} \underline{u}_{r}\right)\right) \\
& \quad-\left(\alpha_{1} \alpha_{2}+\left(\frac{\alpha_{1} Y_{1}}{\rho_{2} \kappa_{2}}-\frac{\alpha_{2} Y_{2}}{\rho_{1} \kappa_{1}}\right)\left(Y_{1}-\alpha_{1}\right)\right) \underline{u}_{r} . \nabla p
\end{aligned}
$$

We use again a fractional step method to solve this system. First, a "dissipative advection step" is performed, which consists in solving the following equation

$$
\partial_{t} \alpha_{1} \frac{Y_{1} \alpha_{1} C_{2}-Y_{2} \alpha_{2} C_{1}}{\alpha_{1} C_{2}+\alpha_{2} C_{1}} \underline{u}_{r} . \nabla \alpha_{1}
$$

Considering that $\underline{u}_{a}=\frac{Y_{1} \alpha_{1} C_{2}-Y_{2} \alpha_{2} C_{1}}{\alpha_{1} C_{2}+\alpha_{2} C_{1}} \underline{u}_{r}$ is a "dissipative advection velocity", we use a characteristic method to solve it, which ensures that the volume fraction $\alpha_{1}$ remains between 0 and 1 if it was in this interval before, providing that the $\mathrm{cfl}$ condition $\Delta t \leq \min _{1 \leq i \leq N} \frac{\Delta x}{\left|u_{a i}\right|}$ is verified, which is usually the case. If this is not the case, the time step is reduced and several "dissipative advection steps" are performed until the convective time step is reached.

Next, we focus on the term including the norm of the pressure gradient. We therefore want to solve the equation

$$
\partial_{t} \alpha_{1}=-\varepsilon_{u} \frac{\alpha_{1} \alpha_{2}\left(\rho_{1}-\rho_{2}\right)}{\rho^{2}\left(\alpha_{1} C_{2}+\alpha_{2} C_{1}\right)}\left(\alpha_{1} \alpha_{2}+\left(\frac{\alpha_{1} Y_{1}}{\rho_{2} \kappa_{2}}-\frac{\alpha_{2} Y_{2}}{\rho_{1} \kappa_{1}}\right)\left(Y_{1}-\alpha_{1}\right)\right)\|\nabla p\|^{2}
$$

In this step, we evaluate the pressure gradient explicitly, and then we solve this equation using a Newton method for each point.

Finally, we perform a "dissipative convection step", which consists in solving the system

$$
\begin{aligned}
\rho \partial_{t} Y_{1} & =-\operatorname{div}\left(\rho Y_{1} Y_{2} \underline{u}_{r}\right) \\
\rho \partial_{t} i & =\operatorname{div}\left(\left(h_{2}-h_{1}\right) \rho Y_{1} Y_{2} \underline{u}_{r}\right) \\
\partial_{t} \alpha_{1} & =-\frac{\alpha_{1} \alpha_{2}}{\left(\alpha_{1} C_{2}+\alpha_{2} C_{1}\right)}\left(C_{1} \operatorname{div}\left(Y_{2} \underline{u}_{r}\right)+C_{2} \operatorname{div}\left(Y_{1} \underline{u}_{r}\right)\right)
\end{aligned}
$$

which is done using a Newton method. 


\section{Numerical results}

\subsection{Air-water shock tube}

We present the numerical results for a shock-tube problem, which consists in a tube of length $1.0 \mathrm{~m}$, filled with air and water with a homogeneous volume fraction $\alpha_{1}=0.5$ through the tube. The tube is divided into a high-pressure room for $x<0.7 \mathrm{~m}$, where the pressure is $p=10^{9} \mathrm{~Pa}$, and a low pressure room $(x>0.7 \mathrm{~m})$ where $p=10^{5} \mathrm{~Pa}$. Water and air are both governed by Stiffened gas equations of state, with the following thermodynamical properties: $\gamma_{G}=1.4, p_{G, \infty}=0 \mathrm{~Pa}$, $\gamma_{L}=4.4, p_{L, \infty}=6.010^{8} \mathrm{~Pa}$. The velocity relaxation time is assumed to be a constant. To determine it, we require that the drag force we use must be equal to the drag force used in the Neptune code [1], which gives the equality

$$
\left.\underline{M}_{1}^{d}=\frac{\rho}{\varepsilon_{u}} \underline{u}_{2}-\underline{u}_{1}\right)=\frac{1}{8} S_{V} \rho_{2} C_{D} \underline{u}_{2}-\underline{u}_{1} \mid\left(\underline{u}_{2}-\underline{u}_{1}\right)
$$

with $S_{V}=\frac{\alpha_{1} S_{\text {bubble }}}{V_{\text {bubble }}}=\alpha_{1} \frac{3}{R}$ is the interfacial area per volume unit and $C_{D}$ is the drag coefficient, given by $\frac{24}{R e}\left(1+0.1925 R e^{0.63}\right)$ [8], with the Reynolds number $R e=\frac{\alpha_{2} \rho_{2} 2 R\left|\underline{u}_{2}-\underline{u}_{1}\right|}{\mu_{2}}$. We take the dynamic viscosity of water $\mu_{2}=10^{-3} \mathrm{~Pa}$.s, and we choose the bubble radius as $R=510^{-4} \mathrm{~m}$, and the remaining variables are found by performing single-phase shock-tube test case for water and air, which give $\rho_{\text {air }}=0.47 \mathrm{~kg} \cdot \mathrm{m}^{-3}, \rho_{\text {water }}=1100 \mathrm{~kg} \cdot \mathrm{m}^{-3}, u_{\text {air }}=25000 \mathrm{~m} \cdot \mathrm{s}^{-1}$ and $u_{\text {water }}=230 \mathrm{~m} . \mathrm{s}^{-1}$. Thus, it is found that $\varepsilon_{u}$ is equal to $2.410^{-5} \mathrm{~s}$.

On Figure 1, we compare results obtained with the five-equation reduced model of [3], the seven-equation model and the present dissipative model. The solution consists in a rarefaction, a contact discontinuity and a shock traveling to the right: the initial pressure imbalance pushes a plug of water to the right (the front is the shock wave, the back is the contact discontinuity) and a low-pressure zone appears in the wake (the rarefaction wave). It is seen that the addition of dissipative terms allows to reproduce to a large extent the results of the seven equation one while the non-dissipative model is unable to introduce any velocity disequilibria between the two phases. We also note that the seven equation model predicts a change in the mass fraction inside the shock zone, this feature is reproduced by the dissipative model whereas the reduced model of [3] is unable to reproduce this feature.

\subsection{Water-faucet problem}

We now perform the water faucet test, which is a usual benchmark test for twophase flows [9]. It consists in a vertical column of length $12 \mathrm{~m}$, where a column of water is surrounded by air. Initially, the mixture is homogeneous along the tube, where the air volume fraction is $\alpha_{1}=0.2$, air density and velocity are $\rho_{1}=$ $1 \mathrm{~kg} . \mathrm{m}^{-3}$ and $u_{1}=0 \mathrm{~m} . \mathrm{s}^{-1}$, water density and velocity are $\rho_{2}=1000 \mathrm{~kg} \cdot \mathrm{m}^{-3}$ and $u_{2}=10 \mathrm{~m} \cdot \mathrm{s}^{-1}$, and pressure is equal to $p=10^{5} \mathrm{~Pa}$. We again use the stiffened 

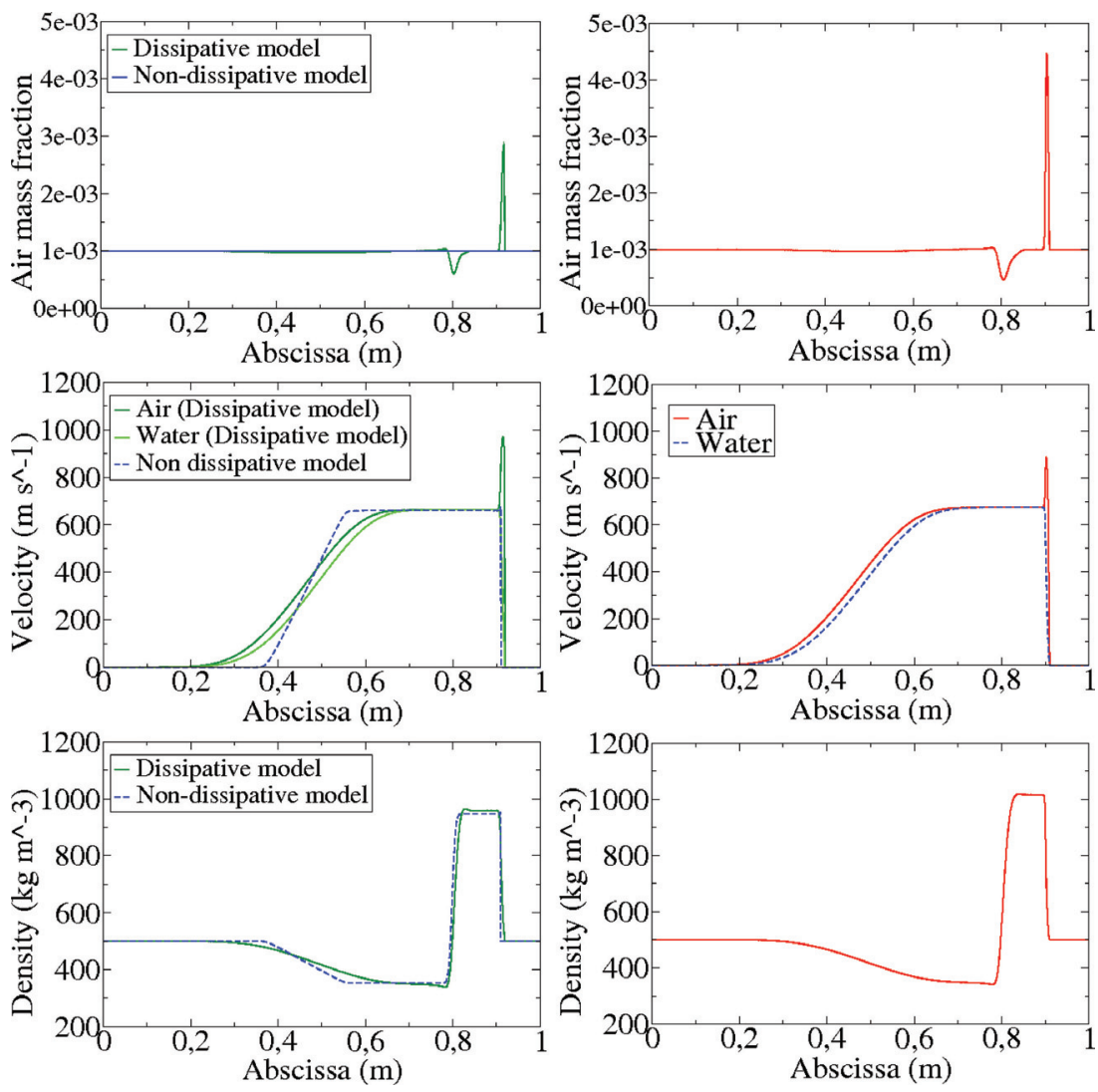

Figure 1: Comparison between the reduced model of [3] and the dissipative model (left) and the seven-equation model (right): air-water shock tube test after $150 \mu$ s. Mesh of 2001 nodes.

gas equation of state for both phase, with the same thermodynamic properties as in the previous test.

At time $t=0 \mathrm{~s}$, a gravity field with $g=9.81 \mathrm{~m} \cdot \mathrm{s}^{-2}$ is applied, which causes a lengthening of the water jet. This test is interesting because air and water have different velocities, and therefore the five-equation model completely fails to reproduce results given by the six- or seven-equation models. Figure 2 shows the results for the dissipative five-equation model and the seven-equation model on a mesh of 1001 nodes. The velocity relaxation time has been fixed to $\varepsilon_{u}=10^{3} \mathrm{~s}$, because this test is usually performed with no drag force, and although we cannot have zero drag in this five-equation model, such a value will reduce it dramatically. It should be noted that this values is also used for the seven-equation model. The pressure relaxation time is set to $\varepsilon_{p}=10^{-12} \mathrm{~s}$ for the latter model, which ensure an equality of the phase pressures. 

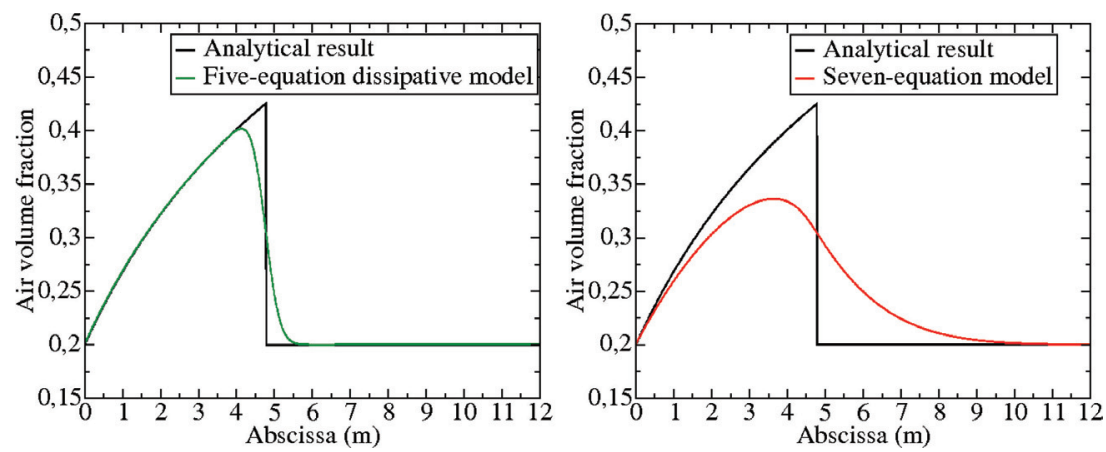

Figure 2: Comparison between the dissipative five-equation model (left) and the seven-equation model (right): Water-faucet test after 0.4 s. Mesh of 1001 nodes.

If we make the approximation of the incompressibility of the liquid and if we neglect the variations of the gas pressures, an analytic solution can be found. It can be seen on Figure 2 that the dissipative five-equation model reproduces in a large extent the variation of the air volume fraction. It can be even noticed that the scheme is less diffusive than the seven-equation model, because of the large number of waves of the latter. Thus, our model can deal with flow phenomena including large velocity differences between the phases, even if it is originally designed for small relative velocities.

\section{Conclusion}

We have derived a five-equation dissipative model from the standard bifluid sixequation model using first order Chapman-Enskog expansions. Thanks to these dissipative terms, this model can deal with flows featuring differences between phase velocities, although it features only one velocity. Numerical results are very promising, and show that the model can simulate flows with shocks or countercurrents in an accurate way, while being hyperbolic and less complex than the two-pressures two-velocity models.

\section{Acknowledgements}

The work of Mathieu Labois and Marc Grandotto has been achieved in the framework of the NEPTUNE project, financially supported by CEA (Commissariat à l'Énergie Atomique), EDF (Électricité de France), IRSN (Institut de Radioprotection et de Sûreté Nucléaire) and AREVA-NP. 


\section{References}

[1] Guelfi, A., Bestion, D., Boucker, M., Boudier, P., Fillion, P., M., G., Hérard, J.M., Hervieu, E. \& Péturaud, P., Neptune - a new software platform for advanced nuclear thermal-hydraulics. Nuclear Science and Engineering, 156(3), pp. 281-324, 2007.

[2] Baer, M. \& Nunziato, J., A two-phase mixture theory for the deflagrationto-detonation transition in reactive granular materials. Journal of Multiphase Flows, 12, pp. 861-889, 1986.

[3] Murrone, A. \& Guillard, H., A five equation reduced model for compressible two-phase flow computations. Journal of Computational Physics, 202(2), pp. 664-698, 2005.

[4] Natalini, R., Recent mathematical results on hyperbolic relaxation problems. Analysis of systems of conservation laws, Chapman and Hall/CRC, Boca Raton, FL: Aachen, pp. 128-198, 1997.

[5] Drew, D. \& Passman, S., Theory of multicomponents fluids. Springer: New York, 1998.

[6] Guillard, H. \& Duval, F., A darcy law for the drift velocity in a two-phase flow model. Journal of Computational Physics, 224(1), pp. 288-313, 2007.

[7] Buffard, T., Gallouet, T. \& Hérard, J.M., A sequel to a rough godunov scheme: Application to real gases. Computers and fluids, 29, pp. 673-709, 2000.

[8] Clift, R., Grace, J. \& Weber, M., Bubble drops and particles. Academic Press: New York, 1978.

[9] Saurel, R. \& Abgrall, R., A multiphase godunov method for compressible multifluid and multiphase flows. Journal of Computational Physics, 150, pp. 425-467, 1999. 